\title{
Genius Math: Uma Aplicação Mobile para Auxiliar a Aprendizagem da Matemática na Pré-escola
}

\author{
Stefane V. Menezes ${ }^{1}$, Jiani C. da Roza ${ }^{1}$ \\ ${ }^{1}$ Instituto Federal Farroupilha - Campus Alegrete (IF Farroupilha) \\ Caixa Postal 118 - 97.555-000 - Alegrete - RS - Brazil \\ Stefanemenezes0@gmail.com, jiani.rozadiffarroupilha.edu.br
}

\begin{abstract}
This article presents an application of m-learning called Genius Math aimed to support the teaching and learning of mathematics in early childhood education. When we consider that in early childhood education learning many are given from the curiosity and enthusiasm of children, the application developed in the Java programming language and geared Mobile Android operating system aims to explore the knowledge of numbers geometric shapes and initiating the operations of addition and subtraction supported by pictures and sounds. In this way, through playing activities for children and activities planned by the teacher with the use of digital technologies can enable contemporary pedagogical practices to children everyday. To test the application was made an interaction test in the classroom for children of early childhood education that in randomly formed groups, they played with different mathematical activities proposed in the application.
\end{abstract}

Resumo. Este artigo visa apresentar uma aplicação de m-learning denominado Genius Math voltado a apoiar o ensino e a aprendizagem da matemática na educação infantil. Ao considerarmos que na educação infantil muitas aprendizagens se dão a partir da curiosidade e do entusiasmo das crianças, o aplicativo desenvolvido na linguagem de programação Java e voltado para celulares com sistema operacional Android busca explorar o conhecimento dos números, formas geométricas e a iniciação as operações de adição e subtração apoiado em figuras e sons. Desta forma, através de atividades lúdicas para as crianças e de atividades planejadas pelo professor com o uso das tecnologias digitais pode-se viabilizar práticas pedagógicas contemporâneas ao cotidiano infantil. Para testar o aplicativo foi realizado um ensaio de interação em sala de aula por crianças da educação infantil que, em grupos formados aleatoriamente, brincaram com as diferentes atividades matemáticas propostas no aplicativo.

\section{Introdução}

As Tecnologias de Informação e Comunicação (TIC) tem oportunizado às pessoas formas cada vez mais dinâmicas e atrativas de comunicação e interação social. Aos poucos estamos abandonando nossa condição passiva frente às diversas atividades $\mathrm{e}$ serviços cotidianos realizados, com facilidade em um curto espaço de tempo e lugar através de uma tecnologia digital tal como o: celular, tablet ou computador. 
V Congresso Brasileiro de Informática na Educação (CBIE 2016)

Anais do XXVII Simpósio Brasileiro de Informática na Educação (SBIE 2016)

Segundo uma pesquisa realizada pelo IBGE (2010) sobre o uso de tecnologias da informação pelos brasileiros, o acesso à internet pelo celular ultrapassou o acesso à internet pelo computador. Neste sentido, reconhecendo o potencial dos dispositivos móveis na atualidade para interação social, serviços e entretenimento e, sendo o celular uma mídia com capacidade de penetração social ampla, basicamente amparada na comunicação oral e de custo acessível, torna-se oportuno explorar cada vez mais a utilização do celular no âmbito educacional.

O conjunto de atividades a serem exploradas no Genius Math toma por base as atividades matemáticas apresentadas nos materiais do projeto Buriti Mirim 2 para a Educação Infantil da editora Moderna. O projeto Buriti Mirim é adotado em algumas instituições nacionais com oferta de pré-escola, sendo assim, ao identificarmos que uma instituição da cidade de Alegrete também o utilizava como material de apoio pedagógico e considerando que o mesmo contempla em seus diferentes eixos de aprendizagem a matemática optamos por utilizá-lo como estratégia de ensino.

A atividade lúdica é fundamental para subsidiar o processo de construção do conhecimento e por isso é indispensável na prática educativa [SANTOS, 2011]. Nesta intenção, as atividades desenvolvidas no aplicativo são realizadas considerando a necessidade das crianças de aprenderem brincando, neste caso brincando no celular, com figuras e formas geométricas.

Genius Math está desenvolvido em Java e é um aplicativo para celulares com sistema operacional Android. O desenvolvimento do aplicativo foi acompanhado por duas professoras da educação infantil que enfatizaram a necessidade de brincar e explorar os números cardinais e as operações aritméticas de adição e subtração. Após o desenvolvimento, o aplicativo foi submetido a uma experimentação através de testes de interação com o usuário, neste caso crianças da educação infantil de 5 a 6 anos.

\section{O Genius Math na perspectiva de um aplicativo educacional}

Pesquisas realizadas mostram que há um constante crescimento do uso da TIC no Brasil no âmbito educacional [DE SOUZA MONTEIRO et al., 2015]. Da mesma forma, é cada vez mais comum o acesso a aplicativos educacionais disponibilizados através de dispositivos móveis, conhecidos vulgarmente por Apps, um forte representante das TIC atuais. A interação através de dispositivos móveis traz formas de interação mais dinâmicas e capazes de expandir as relações professor-estudante e estudante-estudante dentro e fora das escolas, uma vez que a interação pode alterar as relações tanto no tempo (síncrona e assíncrona) [HRASTINSKI, 2008] quanto no espaço (presencial e a distância) [NORTVIG, 2014].

Neste sentido, o aprendizado móvel (ou simplesmente m-learning) pode ser entendido como uma parte específica do aprendizado eletrônico (e-learning) para dispositivos móveis [GEORGIEV et al., 2004]. O m-learning se popularizou a partir do barateamento e acesso facilitado aos dispositivos móveis, dentre eles os celulares que hoje disponibilizam diversos recursos integrados tais como câmera, GPS, tela sensível ao toque e principalmente acesso à Internet, facilitando a obtenção de aplicativos para diferentes finalidades por qualquer usuário. 
V Congresso Brasileiro de Informática na Educação (CBIE 2016)

Anais do XXVII Simpósio Brasileiro de Informática na Educação (SBIE 2016)

Desta forma, o Genius Math é uma das diversas iniciativas de utilizar a tecnologia digital, já presente no dia a dia das crianças, com um fim educativo e de entretenimento, pois a criança aprende brincando e interagindo com as outras.

\section{Trabalhos Relacionados}

Visando coletar informações de layout, conteúdo e abrangência, entre outras características a serem consideradas no Genius Math, foi conduzida uma busca sistemática na PlayStore do Google, por dois motivos: primeiramente por ser uma loja virtual que disponibiliza o download gratuito a Apps para um acervo de aplicativos que podem ser consultados/utilizados por qualquer usuário de celular, inclusive crianças, que com poucos cliques realizam o download. A busca foi refinada em diversas formas: (1) Categoria - Apps educativos, Subcategorias - Apps gratuitos, matemática para crianças, em todas as classificações (número de estrelas de classificação que o aplicativo recebe dos usuários); (2) Categoria - Educativos, Subcategorias - em todas as classificações; (3) Categoria - Jogos e Apps populares, para crianças até 5 anos de idade (uma vez que a outra categoria disponível oferece para crianças de 6 a 8 anos de idade).

Nas três buscas realizadas com diferentes refinamentos foi possível perceber que os Apps resultantes da primeira categoria estavam mais próximos do que se pesquisava, uma vez que nas demais categorias já pré-configuradas na PlayStore retornavam aplicativos para diferentes aprendizagens em física, português, inglês, entre outros. Assim, no primeiro grupo retornaram mais de oitenta aplicativos, porém muitos deles não estabeleciam a faixa etária atendida apenas a finalidade no contexto de ensino e aprendizagem da matemática. Desta forma, foi necessário clicar na descrição desses aplicativos para avaliar os que estivessem próximos a proposta do Genius Math e que na descrição se dizia voltado ao aprendizado da matemática na pré-escola ou educação infantil. Assim, foram analisados os seguintes aplicativos:

Soma para crianças: Seu objetivo é a realização de cálculos de adição. Suas opções de jogos são controladas por tempo, sendo possível a escolha entre um ou dois minutos para a realização dos cálculos, o usuário deve realizar o maior número possível de cálculos no tempo determinado, quando este acaba são informados quantos resultados positivos e negativos o usuário fez. Em um teste rápido foi possível perceber que o maior resultado possível seria oito, pois os cálculos são relacionados às imagens e estas não suportam mais do que quatro elementos em cada, além do fato de todos os feedbacks serem em inglês.

Educational Math for Kids: São apresentados cálculos de adição e subtração para o usuário e este deve selecionar o resultado correto, não existe uma quantidade máxima de cálculos a serem resolvidos, porém o usuário tem três chances de acertar o resultado correto, caso não o faça, é informado que o jogo acabou e o jogo é iniciado novamente. Há a possibilidade de aumentar a dificuldade através das três modalidades em que o aplicativo divide-se. Verificou-se que não é informado para o usuário previamente que o jogo tem um controle de tentativa e erro, onde o usuário tem três chances de erro antes do jogo ser reiniciado, também pode-se citar o fato de que não há um número máximo de cálculos à serem realizados e por este motivo, muitas vezes os cálculos se repetem, além de que o sistema é em inglês e o seu nível de dificuldade requer que o usuário já tenha certo conhecimento da matemática, pois em todos os seus níveis são apresentados 
V Congresso Brasileiro de Informática na Educação (CBIE 2016)

Anais do XXVII Simpósio Brasileiro de Informática na Educação (SBIE 2016)

cálculos que resultam em números negativos. Na primeira utilização do aplicativo foi sugerida a migração para a sua versão paga e foi avisado que esta versão estava obsoleta, ao fim deste estudo a versão grátis já não estava mais disponível na PlayStore.

Matemática para crianças Soma: conhecido como Matemática das maçãs, em sua descrição na PlayStore menciona seu benefício às crianças em fase de aprendizado inicial da matemática. Tem como foco a operação de adição e como objetivo a resolução de cálculos simples que resultem no máximo em dez. São apresentados para as crianças valores e o resultado de zero até dez, todos os números tem representações de maçãs em seus respectivos valores, tem total interação de voz em português. Um fato observado esta aplicação é a demora entre os cálculos e durante sua execução, o que pode fazer com que as crianças percam o interesse ao ter que esperar.

Nos aplicativos analisados foi notada a falta de níveis diferentes de aprendizagem nas atividades disponíveis. Desta forma, o Genius Math busca incluir em suas atividades diferentes níveis de dificuldade. A tabela 1 mostra um comparativo dos aplicativos analisador e do aplicativo Genius Math.

Tabela 1: Comparação entre os softwares disponíveis e o Genius Math

\begin{tabular}{|c|c|c|c|c|c|c|}
\hline & $\begin{array}{c}\text { Idioma } \\
\text { Português }\end{array}$ & $\begin{array}{c}\text { Diferentes } \\
\text { níveis de } \\
\text { dificuldade }\end{array}$ & $\begin{array}{c}\text { Direcionado } \\
\text { a crianças da } \\
\text { pré-escola }\end{array}$ & Música & $\begin{array}{c}\text { Letra } \\
\text { maiúscula }\end{array}$ & Gratuito \\
\hline $\begin{array}{c}\text { Soma para } \\
\text { crianças }\end{array}$ & & $\checkmark$ & $\checkmark$ & & & $\checkmark$ \\
\hline $\begin{array}{c}\text { Educational } \\
\text { math for kids }\end{array}$ & & $\checkmark$ & & & $\checkmark$ & \\
\hline $\begin{array}{c}\text { Matemática } \\
\text { para crianças } \\
\text { Soma }\end{array}$ & $\checkmark$ & & $\checkmark$ & & & $\checkmark$ \\
\hline Genius math & $\checkmark$ & $\checkmark$ & $\checkmark$ & $\checkmark$ & $\checkmark$ & $\checkmark$ \\
\hline
\end{tabular}

FONTE: Adaptado de FEITOSA (2001).

\section{O aplicativo Genius Math}

O aplicativo Genius Math tem como intuito proporcionar a possibilidade do conhecimento junto ao entretenimento. Com foco na matemática da pré-escola, o aplicativo disponibiliza dois módulos distintos, sendo um deles com foco na quantificação e o outro em operações aritméticas de adição e subtração, usando ilustrações para facilitar o entendimento das atividades.

\subsection{Metodologia utilizada}

A proposta de implementar um aplicativo com fins educacionais para apoiar a aprendizagem da matemática surgiu a partir de uma pesquisa exploratória que segundo GIL (2002) visa proporcionar maior familiaridade com o problema. Desta forma, uma pesquisa exploratória foi realizada visando identificar o uso das tecnologias digitais nas séries iniciais e educação infantil em algumas escolas da cidade. Percebeu-se que boa parte das escolas levam as crianças até os laboratórios de informática, semanalmente ou 
V Congresso Brasileiro de Informática na Educação (CBIE 2016)

Anais do XXVII Simpósio Brasileiro de Informática na Educação (SBIE 2016)

quinzenalmente para acessar sítios infantis com diferentes finalidades de aprendizagem. Porém, o uso de aplicativo em celular como instrumento de aprendizagem não foi identificado nas escolas visitadas.

Com estes dados, partiu-se para a busca de materiais para conceder o apoio pedagógico necessário para a criação de um protótipo adequado para a educação infantil. Percebeu-se com as visitas realizadas que algumas escolas adotavam como material pedagógico de apoio os livros do projeto Buriti Mirim [EDITORA MODERNA, 2015]. Decidimos então, utilizar tais livros como fonte pedagógica para criação das imagens (figuras de animais, formas geométricas, números, frutas) e das atividades, com o diferencial que a interação com o aplicativo se dá através do toque, do colorir passando o dedo sobre uma imagem, do apagar ao tocar no cancelar, etc. Nas diferentes atividades disponibilizadas no Genius Math foi utilizado o mínimo de texto possível pois boa parte das crianças de quatro a seis anos ainda não são totalmente alfabetizadas. Também por esse motivo, acreditamos que o Genius Math é um aplicativo educacional para ser utilizado como o apoio do professor ou dos pais, pelo menos até o reconhecimento do aplicativo por parte das crianças.

A aplicação foi desenvolvida para a plataforma Android por vários fatores: a) familiaridade das crianças com o uso de celulares; b) possibilidade de utilizada do App off-line após o download; c) o desejo da desenvolvedora de trabalhar com aplicações móveis para Android. Neste sentido foram utilizadas as seguintes ferramentas: Android Studio, Java, BALSAMIQ e Astah UML.

O desenvolvimento do aplicativo seguiu o processo interativo de geração de modelos a partir da abordagem de prototipação evolutiva conforme definições de Presmann (2011). Quanto a arquitetura e modelagem do sistema o mesmo foi desenvolvido a partir de dois casos de usos principais guiando-se pelos conceitos de Fowler (2014) para modelagem de sistemas orientados a objetos. Desta forma, temos: 1) "contar figuras" e 2) "calcular figuras". O "contar figuras" pode ser estendido pelos casos de usos Selecionar número e pintar figura. Já o caso de uso "calcular figura" inclui o caso de uso "calcular número". Um caso de uso "mostrar recompensa" pode expandir qualquer caso de uso.

Cada atividade (caso de uso) possuiu uma classe Java para a sua configuração que determina, através de métodos, quais serão as imagens e os valores utilizados. Para a realização da recompensa visual foi necessário a inserção de um arquivo $\mathrm{xml}$ com todas as imagens necessárias para a animação. A recompensa visual está presente ao final de cada atividade concluída com sucesso, para que isto acontecesse foi necessária a codificação de uma classe Java denominada Controlador.

\subsection{Desenvolvimento}

Na Figura 1 é apresentado a tela inicial da aplicação Genius Math, no detalhe 1, há um botão que controla a música tocada na aplicação, esta opção pode ser desabilitada em qualquer momento da sua utilização, pois é apresentada em todas as telas. 
V Congresso Brasileiro de Informática na Educação (CBIE 2016)

Anais do XXVII Simpósio Brasileiro de Informática na Educação (SBIE 2016)

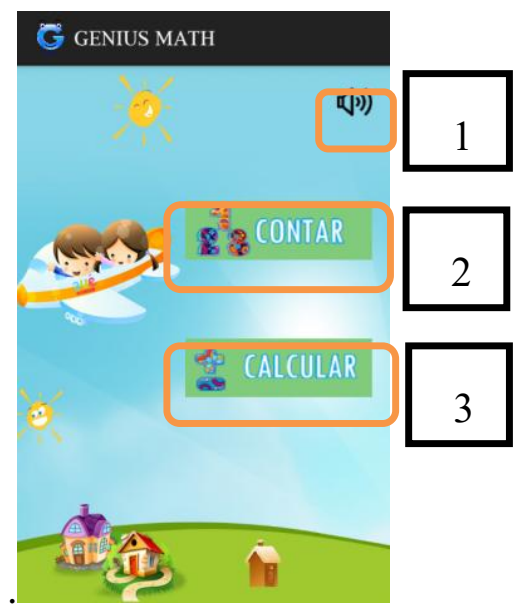

Figura 1. Tela inicial da aplicação Genius Math.

Na Figura 2, à direita, é apresentado o resultado do clique no botão Contar e à esquerda é apresentado o resultado do clique no botão Calcular, apresentados nos detalhes 2 e 3 da Figura 1. Há três atividades disponíveis no módulo Contar, denominados: Contar animais; Contar Colorindo; Contar Formas Geométricas. E três atividades disponíveis no módulo Calcular, denominados: Adição; Subtração; Calculando com formas geométricas.

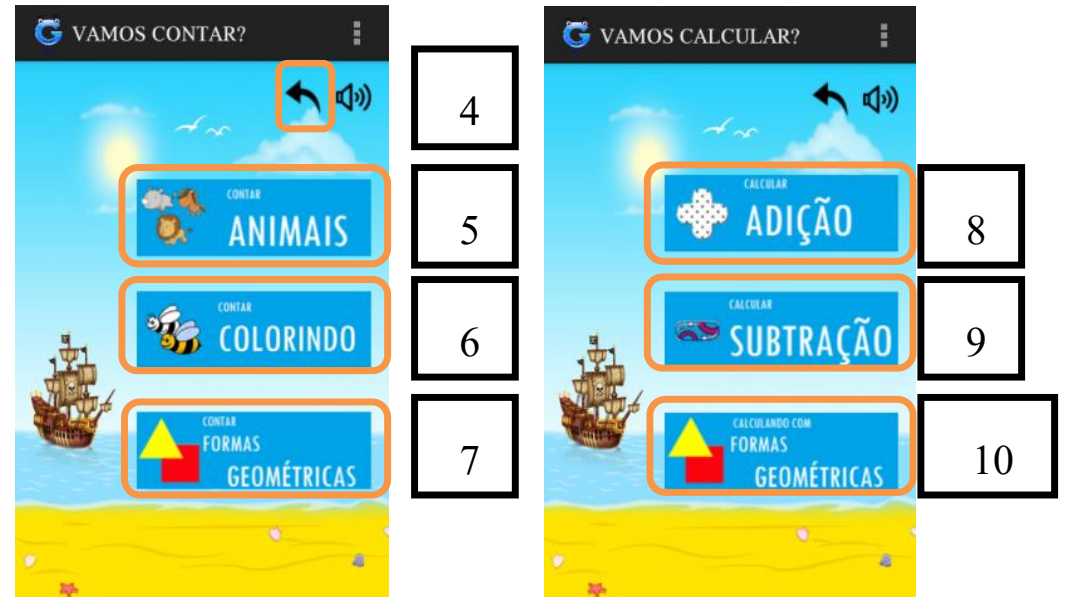

Figura 2. Tela resultado do clique no botão Contar ou Calcular.

No detalhe 4 é ilustrado o botão voltar, apresentado em todas as demais telas, proporcionando a possibilidade de voltar para a tela anterior, quando solicitado. $\mathrm{O}$ detalhe 5, Figura 2, quando clicado, destaca a atividade Contar Animais referente a Figura 3, à esquerda, essa atividade consiste na apresentação de uma imagem com determinados números de animais e três opções de respostas, sendo destas somente uma correta, ao clicar em um botão com o resultado errado o mesmo desaparece, uma mensagem é apresentada para o usuário e um som associado ao erro é emitido. Quando o resultado correto é selecionado é apresentada uma nova imagem, novas opções de resultados e é emitido um som para ser associado ao acerto.

Ao clicarmos no detalhe 6 da Figura 2, denominado Contar Colorindo, é apresentado para o usuário a tela ilustrada na Figura 3, ao centro, nesta atividade são apresentadas nove imagens de animais em preto e branco e a instrução de quantos animais deverão ser pintados, ao clicar em cima de qualquer uma das imagens a mesma 
V Congresso Brasileiro de Informática na Educação (CBIE 2016)

Anais do XXVII Simpósio Brasileiro de Informática na Educação (SBIE 2016)

fica colorida e se selecionada novamente volta ao estado inicial. Se for informado um resultado diferente do solicitado e o botão Continuar, em destaque, for clicado é apresentado uma mensagem para o usuário e não ocorre transição de telas até que o resultado correto seja selecionado.

Quando clicado no detalhe 7 da Figura 2, a atividade Contar Formas Geométricas é solicitada, ilustrada na Figura 3, à direita. Esta atividade consiste na apresentação de uma imagem com determinadas quantidades de triângulos, quadrados e círculos, três opções de resultados e a imagem de qual forma geométrica está sendo solicitada. $\mathrm{O}$ reconhecimento das formas geométricas faz parte da metodologia ensinada na matemática e é explorada pelo aplicativo.
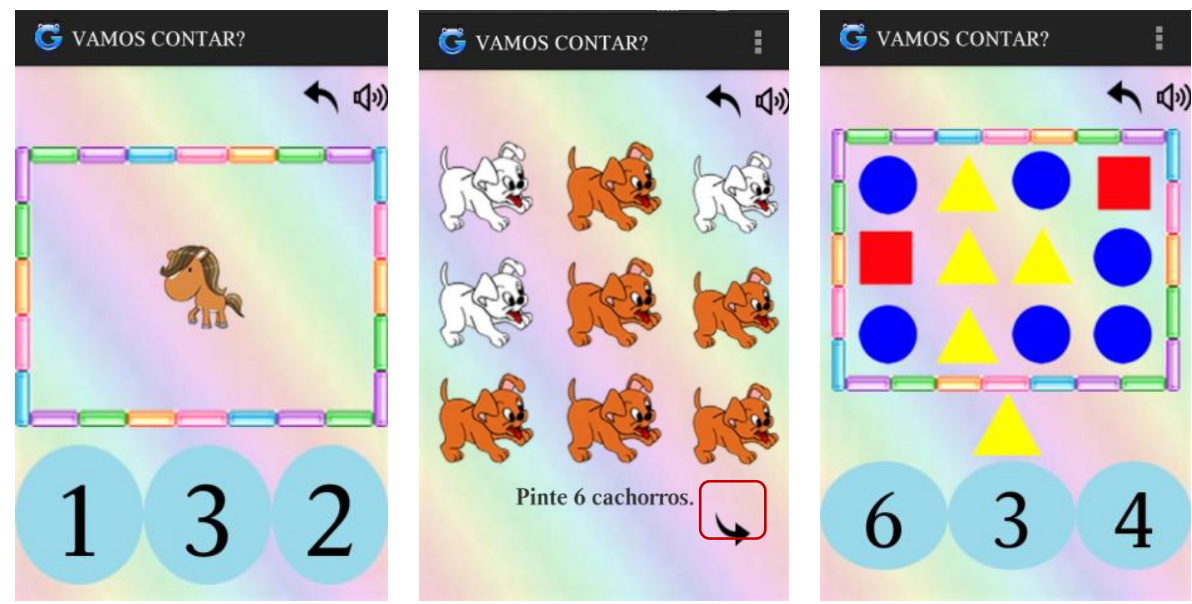

Figura 3. Atividade do módulo Contar

O detalhe 8, apresentado na Figura 2 à direita, se selecionado ilustra a atividade Adição, onde são apresentados pequenos cálculos exclusivamente desta operação, com resultados inferiores ao número dez. A Figura 5, à direita, exemplifica esta atividade, mostrando a utilização de animais para ilustrar os cálculos, além disso os cálculos são montados com algoritmos para melhor entendimento do usuário. São disponibilizados três resultados, sendo destes somente um correto, quando um resultado errado for selecionado o botão torna-se invisível, é emitido o som referente ao erro e uma mensagem é apresentada para o usuário. Ao selecionar o resultado correto é apresentado outro cálculos com novos resultados e um som é emitido para ser associado ao acerto.

O detalhe 9 apresentado na Figura 2, destaca o botão que solicita a atividade exclusiva da operação subtração, quando clicado. Esta atividade contém a mesma metodologia utilizada na atividade de Adição, e é ilustrada na Figura 4, ao centro. Nesta atividade nenhum dos cálculos solicitados tem valor maior que seis, pelo fato de que o público alvo deste aplicativo são crianças que estão na pré-escola e ainda não possuem um domínio claro da operação de subtração.

No detalhe 10 da Figura 2, podemos observar a atividade intitulada Calculando com Formas Geométricas, nesta atividade as operações matemáticas de adição e subtração são trabalhadas juntamente. O intuito desta metodologia é a distinção entre as formas geométricas e o raciocínio lógico necessário para realizar cálculos a partir destas formas. Como observamos na Figura 4, à direita, seu layout e suas funcionalidades são parecidos com as demais atividades explicadas anteriormente. 
V Congresso Brasileiro de Informática na Educação (CBIE 2016)

Anais do XXVII Simpósio Brasileiro de Informática na Educação (SBIE 2016)

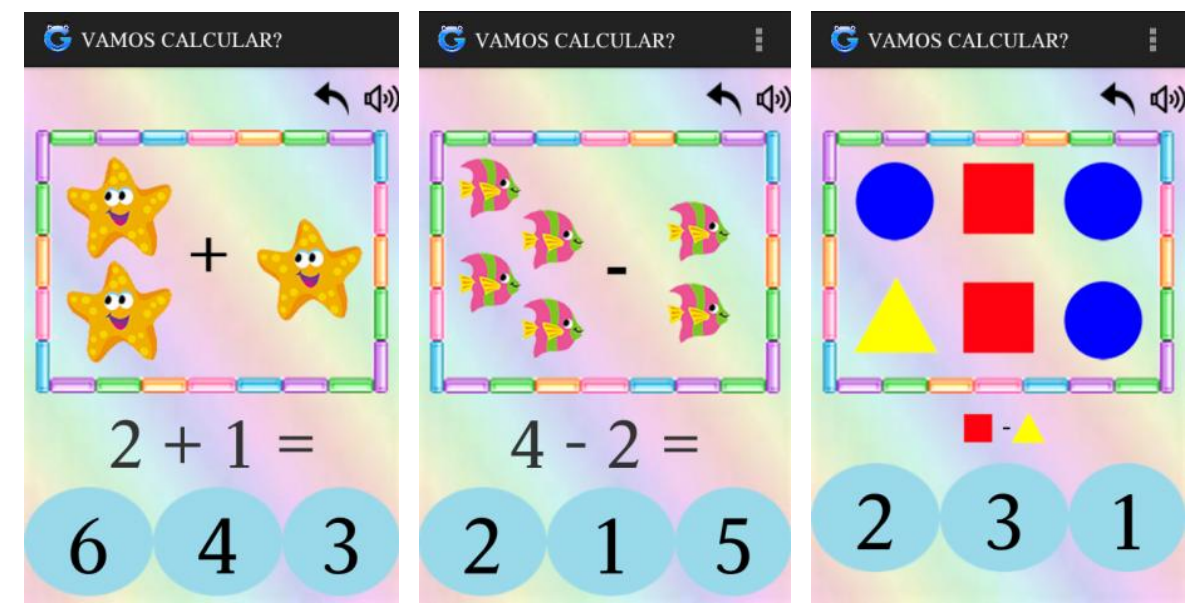

Figura 4. Atividades do módulo Calcular.

A Figura 5 traz as telas de recompensa visual e auditiva apresentada para o usuário a cada ciclo concluído de qualquer uma das atividades apresentadas anteriormente. Consiste em uma animação acompanhada do som de palmas. A mensagem que lhe é apresentada é modificada dependendo da sua quantidade de erros durante a execução da atividade. Caso a criança conclua as atividades com cem por cento de acertos lhe é apresentado a mensagem "VOCÊ É UM GÊNIO", se ele obtiver um número inferior a três erros lhe é apresentado a mensagem "PARABÉNS", se a margem de erro da criança estiver entre quatro e sete erros a mensagem é "LEGAL", estes exemplos podem ser observados na Figura 5. As atividades têm em média dez exercícios cada, por este motivo foi escolhido estes parâmetros para serem utilizados nas mensagens, porém em atividades que tenham menos exercícios estes parâmetros foram adaptados.

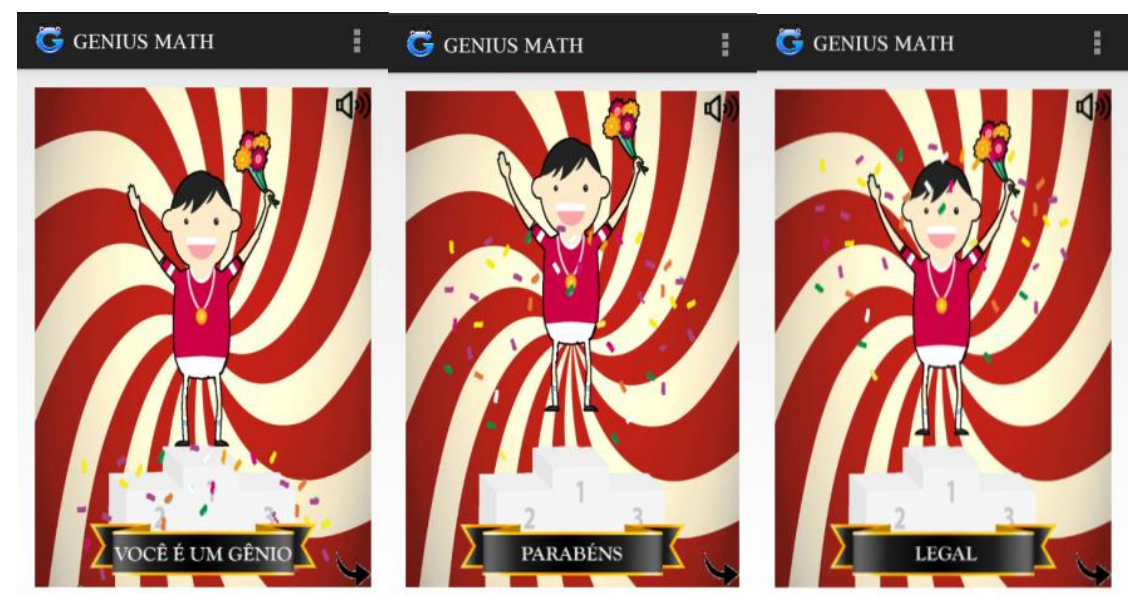

Figura 5. Tela recompensa apresentada ao final das atividades.

\section{Avaliação Experimental}

Com a primeira versão do aplicativo funcional foi realizada a primeira avaliação com uma professora da educação infantil a partir do uso da ferramenta diretamente em um celular. A partir das sugestões da professora, foram realizadas modificações, como por exemplo, o tamanho da fonte e a preferência da utilização de letra maiúscula nos enunciados das atividades e o grau de dificuldade das atividades, onde os cálculos de adição deveriam ter maior grau de dificuldade do que os cálculos de subtração. 
Após as modificações concluídas foi realizada uma segunda avaliação que consistiu em um ensaio de interação [NIELSEN, 1999] onde o aplicativo foi apresentado para o seu público alvo, com o intuito de observar a viabilidade do aplicativo em um ambiente real e detectar possíveis falhas ou ajustes a serem realizados. O aplicativo foi apresentado para vinte estudantes do terceiro ano da pré-escola de uma escola particular situada no centro da cidade de Alegrete. As crianças com idade entre 5 e 6 anos já desenvolviam as noções de matemática apresentadas no aplicativo através do livro Buriti Mirim e outras atividades lúdicas realizadas pela professora.

Para o ensaio de interação as crianças foram organizadas em duplas ou trios para realizarem as atividades, em cada dupla ou trio tinha pelo menos uma criança alfabetizada. Esta didática foi escolhida pela professora, pois as crianças já estão habituadas a este método. Inicialmente notou-se que as crianças que ainda estão em fase de alfabetização tiveram receio ao iniciar o jogo, tendo em vista as nomeações das atividades, e estas crianças escolhiam as atividades pelas imagens contidas nos botões.

Pode ser observado que durante a utilização da atividade Contar Colorindo, a maioria das crianças selecionavam todas as imagens para depois ler o enunciado e objetivar a resposta correta, também se percebeu que as crianças tinham dificuldade em dar continuidade após selecionar as imagens, então se achou necessário mudar a imagem utilizada no botão continuar, para um melhor entendimento das crianças. Os alunos que não sabiam ler tiveram o auxílio dos colegas para ler o enunciado.

A atividade Subtração, por ser uma operação matemática que poucos dominavam, foi uma das atividades em que a maioria das crianças demonstrou menos interesse. Utilizando inicialmente para conhecer a atividade, mas sem interesse em uma segunda utilização.

Algumas crianças ao escolherem a atividade Contar com Formas Geométricas tinham facilidade para entender o objetivo da atividade, outras após o auxílio da professora concluíam a atividade com êxito. Por ser uma atividade onde a instrução é feita a partir de uma imagem, para objetivar quais das formas geométricas estavam em destaque naquele momento, algumas crianças tiveram dificuldades em entender de que a imagem da instrução não deveria ser contada juntamente com a imagem que apresentava as formas geométricas.

Percebeu-se que as crianças após observar que as mensagens apresentadas na recompensa variavam de acordo com a pontuação, fez com que o número de erros diminuíssem. Conclui-se que o aplicativo apresentou resultados satisfatórios mostrandose como uma alternativa viável e de boa aceitação por parte das crianças.

\section{Conclusões e trabalhos futuros}

Esta aplicação oferece a alternativa da utilização da tecnologia tanto dentro de sala de aula quanto fora sendo necessário para a criança apenas um dispositivo mobile para que a utilização do aplicativo seja possível. Diferentemente dos outros aplicativos disponíveis gratuitamente no mercado, o Genius Math oferece variadas atividades e níveis de dificuldades, afim de que a criança sinta-se desafiada a concluir as atividades.

Como trabalhos futuros pretende-se realizar novos ensaios de interação em escolas públicas e em escolas onde o projeto Buriti Mirim não seja empregado com a 
V Congresso Brasileiro de Informática na Educação (CBIE 2016)

Anais do XXVII Simpósio Brasileiro de Informática na Educação (SBIE 2016)

intenção de identificar uma possível adaptação facilitando ao software pelo mesmo utilizar-se como base um material já conhecido das crianças. Também poderão ser implementados novos exercícios nas atividades já existentes. Outra possibilidade em estudo é adaptar a aplicação também para smartphones com o sistema operacional Windows phone, para assim atingir um maior público. E para finalizar intenciona-se disponibilizar o aplicativo gratuitamente na PlayStore.

\section{Referências}

Braga, J. (2015) "Objetos de Aprendizagem: Volume 2 - Metodologias de Desenvolvimento". Santo André: Editora da UFABC.

de Sousa Monteiro, B. et al. (2015). "Youubi: Ambiente de Aprendizagem Ubíqua." In Anais do Simpósio Brasileiro de Informática na Educação. Vol. 26. No. 1.

EDITORA MODERNA. (2015) "Buriti Mirim 2". http://www.moderna.com.br/main.jsp?lumPageId=4028818B2E24D324012E3469E6 0A34AF\&itemId=04F137CC45E1405088B38F5CBD8200F0. Agosto.

Feitosa, T. S. (2001). "Informática na Educação: novas ferramentas pedagógicas para o professor na atualidade." 3 ed. São Paulo: Érica.

Fowler, M. (2014). “UML Essencial: um breve guia para linguagem padrão.” Bookman Editora.

Georgiev, T., Georgieva, E., \& Smrikarov, A. (2004). "M-learning-a New Stage of ELearning." In International Conference on Computer Systems and TechnologiesCompSysTech (Vol. 4, No. 28, pp. 1-4).

Gil, A. C. (2002) "Como elaborar projetos de pesquisa”. São Paulo, 5, 61.

Hrastinski, S. (2008). "Asynchronous and synchronous e-learning." Educause quarterly, 31(4):51-55.

IBGE. (2010) “Acesso à Internet e à televisão e posse de telefone móvel celular para uso pessoal”, http://biblioteca.ibge.gov.br/visualizacao/livros/liv93373.pdf, Abril.

Nielsen, J. (1999). Designing we usability: The practice of simplicity. New Rifers Publishing.

Nortvig, M. (2014). "The Change of Time and Space in E-Learning." American Journal of Educational Research, 2(8):612-616.

Pressman, R. S. (2011). "Engenharia de software: uma abordagem profissional." $7^{\mathrm{a}}$ Edição. Ed: McGraw Hill.

Santos, J. O. (2011) “O lúdico na Educação Infantil.”

Weller, M., Pegler, C. A. and Mason, R. (2003) "Putting the pieces together: What working with learning objects means for the educator." In the Proceedings of the Second eLearnInternational World Summit, Edinburgh International Conference Centre, Edinburgh, Scotland. 